\title{
Huge Cystadenocarcinoma of the Ovary Disguising as Degenerating Uterine Fibroid in Pregnancy: Case Report and Literature Review
}

\section{Adedapo Ande}

University of Benin Teaching Hospital

Maradona Isikhuemen ( $\nabla$ maradona4real2002@yahoo.com )

Federal Teaching Hospital, Abakaliki. Nigeria https://orcid.org/0000-0002-3068-3661

Weyinmi Kubeyinje

University of Benin Teaching Hospital

Michael Ezeanochie

University of Benin Teaching Hospital

\section{Case report}

Keywords: Pregnancy, Ovarian tumour, Ovarian cancer, degenerating fibroid, Cyst Adenocarcinoma.

Posted Date: July 8th, 2021

DOI: https://doi.org/10.21203/rs.3.rs-673624/v1

License: (c) (i) This work is licensed under a Creative Commons Attribution 4.0 International License. Read Full License 


\section{Abstract}

Background: Ovarian malignancy is rare in pregnancy and constitutes $3-6 \%$ of all ovarian tumours associated with pregnancy. It is usually asymptomatic, detected during routine antenatal ultrasound scan. Treatment is mainly surgical. The aim of this report is to share our experience in the management of a referred case of papillary serous cystadenocarcinoma of the ovary disguising as degenerating uterine fibroid in pregnancy. The diagnostic challenges and treatment are highlighted with a view to improving care in our setting.

Case presentation: A 29 year old gravida 2 para 1 with one living child was referred to our unit at 25 weeks gestation with complaint of progressive painful abdominal distension with dyspnoea noticed in the index pregnancy. Ultrasound scans were suggestive of uterine fibroid in pregnancy with degenerative changes for which she had conservative management. Due to worsening symptoms, she had laparotomy and a huge ovarian mass was removed alongside the affected ovary. Histology confirmed Serous Papillary Cyst Adenocarcinoma of the ovary. She is doing well on adjuvant chemotherapy.

Conclusion: A high index of suspicion for ovarian malignancies in pregnancy, coupled with complementing MRI (when available) with ultrasound to differentiate degenerating uterine fibroids is suggested/recommended.

\section{Background}

Ovarian malignancy is uncommon in pregnancy and constitutes of $3-6 \%$ of all ovarian tumours associated with pregnancy. ${ }^{1,2}$ It is the second most reported gynaecological malignancy after cervical cancer in pregnancy. ${ }^{3,4}$ Majority of adnexal masses in pregnancy are functional ovarian cysts which are non-malignant. ${ }^{3,5}$ The incidence of ovarian malignancy in pregnancy appears to be increasing due to routine antenatal ultrasound scan in the evaluation of pregnancy and delay in childbearing. ${ }^{6,7}$ However, pregnancy may complicate diagnosis of ovarian malignancy with ultrasound scan unable to precisely detect an ovarian tumour due to changes in morphology, the quality of the ultrasound machine and the skills of the operator. ${ }^{2,8,9}$ Though ultrasound scan remain primary in evaluating ovarian tumours in pregnancy, magnetic resonance imaging (MRI) is safe and better elucidate both benign and malignant tumours in pregnancy. ${ }^{10,11,12} \mathrm{MRI}$ services are sparse and costly in our practice hence not readily resorted to.

In contrast to ovarian malignancy, uterine fibroids in pregnancy are benign but common and of more burden in our practice due to the preponderance of fibroids in negroids. ${ }^{13.14}$ Fibroids enlarge due to hormones of pregnancy and also undergo degenerative changes; commonly red and cystic degeneration. ${ }^{15,16}$ Ultrasound features to diagnose uterine fibroids in pregnancy, especially with degenerative changes may be misleading. They are often essentially similar to that of ovarian malignancy. ${ }^{7,9}$ Though still primary for evaluating fibroids and other tumours of pregnancy, the quality of USS and operator skills make them inferior to MRI in precise elucidation. ${ }^{11,12}$ Uterine fibroids in 
pregnancy are often asymptomatic and when symptomatic, conservative non-surgical management are often employed effectively. ${ }^{15-17}$ Symptomatic uterine fibroids have been managed by antenatal myomectomy, myomectomy at Caesarean section or obstetric hysterectomy for post partum haemorrhage. ${ }^{18-21}$ Such managements of symptomatic uterine fibroids are consequent to unsuccessful conservative management, dependent on presentation, maternal/foetal effect, decision on delivery and complications.

Despite the observed increase in diagnosis of ovarian malignancy in pregnancy due to delay in childbearing and routine ultrasound scan, the numbers are still too small to prepare standards of treatment, especially in low resource countries. ${ }^{4,6,22}$ The need to balance maternal and foetal/neonatal outcomes further make decisions on best management options in pregnancy challenging. ${ }^{6,22}$ Although current management of ovarian malignancy involve surgical removal, chemotherapy and radiotherapy (singly or in combination), there is no definitive guideline regarding its management in pregnancy. 4,6,22 Evolving consensus suggest that management decisions depend on such factors as the stage, type, gestational age, general condition of mother and foetus and the desire to continue the pregnancy.

It is suggested that ovarian masses in pregnancy be removed if the diameter is more than $10 \mathrm{~cm}$, it persists to the second trimester or show solid or mixed highly suspicious texture during ultrasound. ${ }^{4,23}$ Surgical removal is considered best between 16-20 weeks of gestation because of reduced risk of pregnancy loss amongst other reasons. ${ }^{6,9}$ If possible, delaying surgery till the second or third trimester reduces the chance of miscarriage. ${ }^{24}$ Staging procedure which may include appendectomy, infracolic omentectomy, pelvic peritoneal biopsies or lymph node dissection may not be possible in pregnancy due to lack of access to the pouch of douglas and pelvic peritoneum. In such instances, a restaging is planned postpartum. ${ }^{6,25}$ Optimal treatment of ovarian and other malignancies in pregnancy are better achieved by collaboration of multidisciplinary teams. . $^{4,6,7,22}$

Chemotherapy is associated with complications such as spontaneous abortions, congenital malformations and hypertrophy, which are observed more with multi-agents. ${ }^{26,27}$ If considered, it is recommended from the second trimester onward, after completion of organogenesis and allowing a three-week window from the last course to delivery for reduction of maternal risk of haemorrhage, infection or anaemia and chemo-accumulation in the foetus/newborn. ${ }^{6,22,27}$ Radiotherapy is contraindicated as it may cause foetal death, abnormalities and growth impairment, with predisposition to carcinogenesis during pregnancy or in childhood. ${ }^{28}$ It may only be considered if embryo-foetal death is unavoidable. $^{4}$

Experience in the management of ovarian malignancy in pregnancy particularly in the West African Sub Region is sparse. The aim of this report is to share our experience in the management of a referred case of papillary serous cystadenocarcinoma of the ovary disguising as degenerating uterine fibroid in pregnancy. The diagnostic challenges and treatment are highlighted with a view to improving care in our setting. 


\section{Case Presentation}

A 29 year old gravida 2 para 1 with one living child was referred to our unit from a private hospital at 25 weeks gestation with complaint of progressive abdominal distension noticed in the index pregnancy. There was associated increasing abdominal pain and difficulty with breathing due to the abdominal distension. She was well before the index pregnancy with no abdominal swelling. There was no weight loss and no other history suggestive of gynaecological malignancy. Her delivery about 2 years before presentation was an emergency Caesarean section for failed induction of labour (gestational diabetes was the indication for induction) at the referring centre. The outcome was a $3 \mathrm{~kg}$ male with no abnormality, alive and well. Recourse to the operation notes revealed a fibroid was removed because it was in the line of the lower segment incision and there were some subserous fibroid seedlings. Otherwise, the ovaries and other structures were noted to be normal.

She booked index pregnancy at the referring centre at 12 weeks gestation with no unusual complaints. The uterine size was compatible with date and no abnormal mass was palpable. At her follow-up clinic, she was told the uterine size was slightly larger than her date and she had uterine fibroids. The uterine size increased as the pregnancy progressed and was later associated with abdominal pains. She had series of four ultrasound scans all of which found uterine fibroid with increasing size and degenerative changes (cystic or red degeneration). The foetus was normal, with no abnormality. The abdominal pain progressively worsened with increasing abdominal swelling and difficulty breathing. These necessitated several oral and parenteral medications to relief the pain (including paracetamol, pentazocine, diclofenac, tramadol and dihydrocodeine), nifedipine for tocolysis on out and in-patient basis. Despite these conservative management, her symptoms did not abate, it rather worsened, hence her referral.

At presentation, she was pale (PCV 24\%), dyspnoec (RR 26/min), tachypnoec (PR 128/min), with abdominal pain. She weighed $88 \mathrm{~kg}$ and was $1.66 \mathrm{~m}$ tall with no pedal oedema. Her blood pressure and SPO2 were normal. Symphysiofundal height was $44 \mathrm{~cm}$ and foetal heart was heard and regular. There was irregularity of the uterine outline on the right fundo-lateral portion which was cystic, mobile and markedly tender. A repeat ultrasound showed a viable foetus at 24 weeks gestation with reduced activity and liquor volume. There was a huge subserous fibroid measuring $36 \times 26 \mathrm{~cm}$ located in the right anterolateral aspect of the uterus with cystic degeneration. A diagnosis of viable pregnancy with huge degenerating uterine fibroid and symptomatic anaemia was made.

The couple was counselled on the diagnosis, the need for immediate admission to correct the anaemia by blood transfusion and institution of conservative management. The hope of abatement of her symptoms and allowing the foetus to further mature for successful extrauterine life was explained to them. They were made to understand that conservative management will be terminated by surgical intervention (myomectomy) if her clinical condition worsened. The couple agreed to the planned line of management. She has 2 units of blood transfused and her haematocrit improved to $30 \%$ with less dyspnoea (RR 20/min). Pain relief was initially by parenteral then oral paracetamol, pentazocine and diclofenac and intermittent rectal diclofenac. Hydration was ensured. She was encouraged to take 
suitable positions/postures that made her comfortable, reduce pain and breathe better. Other treatments were: 72 hours intravenous then 7 days oral antibiotics (amoxicilline/clavulanic acid) and oral nifedipine $10 \mathrm{mg} 12$ hourly for tocolysis and haematinics with vitamin C.

She initially responded well to conservative management, but by the 10 th day (i.e 26 weeks and 3 days gestation), the abdominal pain with respiratory embarrassment worsened and became unbearable. The couple consented for emergency laparotomy and myomectomy. The procedure was done under intubation general anaesthesia. Intraoperatively, a $4.5 \mathrm{~kg}(30 \times 25 \mathrm{~cm})$ right ovarian mass which had cystic and solid components was found. The ovarian mass rested completely on the uterus such that there was no separation between the two but there was no attachment between them. The mass was removed alongside the right ovary. The part of the omentum which was attached to the tumour was also excised. The left ovary was grossly normal. There was no ascitis and no tumour deposits were seen in the abdomen. The blood loss at surgery was $1.5 \mathrm{~L}$ and she had 3 units of whole blood transfused. Anaesthesia was reversed and she was returned to the recovery room from where she was subsequently transferred to the ward on antibiotics and analgesics.

Her postoperative recovery was unremarkable. On the second postoperative day, her packed cell volume was $27 \%$. By the 4 th postoperative day, she complained of absent foetal movement and intrauterine foetal demise was confirmed by ultrasound scan. She had vaginal uterine evacuation using misoprostol by the 5 th postoperative day. The foetus was a male $0.85 \mathrm{~kg}$ fresh stillbirth with no obvious abnormality. She was discharged home on the 7th postoperative day. Histology report on the removed ovary and mass was Serous Papillary Cyst Adenocarcinoma. The couple was counselled on the histology report and the need for further management by the Gynaecologic Oncologist. She is currently being followed-up in the Gynaecological Oncology Clinic and doing well on chemoterapy. There is currently no clinical, biochemical or radiological evidence of tumour recurrence.

\section{Discussion}

The rarity of ovarian malignancy compared to the preponderance of uterine fibroids in pregnancy in our practice predispose to occasional clinical challenges differentiating the two and ensuring appropriate and timely management as seen in this case. While antenatal ultrasound has increased identification of ovarian malignancy, the pregnancy hormone effects on uterine fibroids and the degenerative changes may mimic findings in ovarian malignancy. 7,9

The index case had progressive abdominal swelling associated with pain and difficulty in breathing. These are symptoms that may be present in symptomatic ovarian malignancy or uterine fibroid in pregnancy. None of the four ultrasound scans done by specialists suggested ovarian malignancy, but rather uterine fibroid with degenerative changes. Even if the skills of the operator is not in doubt, poor ultrasound machine resolution in the phase of change in morphology in a mass closely abutting on the uterus with no demarcation might have been technically challenging. MRI, usually deployed when ultrasonography is inconclusive was not considered since the ultrasound reports were conclusive of 
uterine fibroids with degenerative changes, a common finding in our practice. Moreso, she had no other symptoms or signs suggestive of a malignancy. Considering the preponderance of uterine fibroids in our practice, complementing MRI with ultrasound should be considered to differentiate degenerating uterine fibroids in pregnancy from ovarian malignancy. Availability and cost are challenges in furtherance of this.

Being a 29 year old primipara, our index patient was in consonance with findings that pregnant women with ovarian malignancy are more likely to be younger than those not pregnant who also tend to be of higher parity. ${ }^{3,25,26}$ Except for these, there were no other risk factors in her. We managed our patient for symptomatic degenerating fibroid in pregnancy, conservatively initially, until worsening of her clinical state necessitated surgery at 26 weeks gestation. It was at the laparotomy that the complex ovarian mass that turned out to be malignant was discovered and removed. Even though uterine fibroids in pregnancy are more common in our practice, a high index of suspicion is recommended in such instance as this to exclude ovarian malignancy. Tumour markers associated with common ovarian malignancies may be affected by pregnancy. ${ }^{26} \mathrm{CA}-125$, a tumour marker associated with ovarian malignancies is elevated in pregnancy, hence it is not reliable in diagnosis. ${ }^{26}$ Serial measurement of CA-125 combined with ultrasound scan has been suggested for the evaluation of ovarian cancer as it is associated with increased sensitivity. ${ }^{29}$ This was however not done for the index patient as ovarian malignancy was not in consideration.

Four days after removal of the ovarian mass, she had ultrasound confirmed intrauterine foetal death at 27 weeks. The fresh still birth had no obvious malformations and the weight was appropriate for age. Though we can not adduce any specific reason for this, we note that surgical removal is considered best at 16-20 weks gestation to reduce risk of pregnancy loss. ${ }^{6,9}$ If the diagnosis was made earlier in pregnancy and the mass removed at the suggested optimal gestation, the foetal outcome might have been better.

Managing ovarian malignancy in pregnancy is challenging. This is more so in our practice as in other low resource countries where late and misdiagnosis may occur as in this case. The numbers of managed ovarian malignancies in pregnancy are still too small to have standardised treatment. ${ }^{4,6,22}$ Sharing experiences and gathering information will facilitate development of standardisation of treatment. The consensus is for multidisciplinary teams collaborating for the optimal treatment of ovarian malignancies. ${ }^{4,6,7,22}$ Although the initial diagnosis was not ovarian malignancy in our patient, the GynaeOncologist and counsellors were involved in her care when the diagnosis was confirmed after her surgery. The Gynae-Oncoloist has continued the care of the patient. Since the management of ovarian malignancies in pregnancy is associated with intense emotional response from the patient and family members, counselling is helpful in reducing the psychological trauma caused by the diagnosis and management. The need to balance foetal/neonatal outcome with maternal care requires the input of neonatologists also.

As with other malignancies, early diagnosis and appropriate treatment of ovarian malignancies in pregnancy offer best prognosis. It has been suggested to remove as soon as possible ovarian masses > 
$10 \mathrm{~cm}$ diameter, enlarging, persisting to second trimester or with highly suspicious mixed texture for histological diagnosis. ${ }^{4,23}$ Our patient did not have the benefit of early removal and histologic diagnosis since the mass was not initially considered ovarian, but fibroid. She did not have the staging procedure; only the well delineated ovary plus mass, and the omentum over it was removed. There was no ascitis or tumour deposits. A re-staging procedure may be planned by the Gynae-Oncologist in the course of her treatment post-partum.

Of surgical removal, chemotherapy and radiotherapy which are current management tenets of ovarian malignancy, surgery and chemotherapy singly, or in combination have pre-eminence in ovarian malignancy in pregnancy. ${ }^{4,6,22}$ Radiotherapy is contraindicated and may only be considered if embryofoetal death is unavoidable. ${ }^{4,28}$ While chemotherapy is associated with some complications, it is worse when started before the second trimester, multi-agents are used or when no sufficient window between last course and delivery. ${ }^{6,27}$ Surgical interventions, especially early, as a means of treatment and histologic diagnosis is presently favoured for ovarian malignancies in pregnancy. 4,6,9,23,25,26 The risk of pregnancy loss after surgery remains a challenge in determining when best and balancing maternalfoetal outcomes. ${ }^{6,9,22}$ Our patient had surgical removal at 26 weeks gestation followed by intrauterine foetal death within the postoperative week.

\section{Conclusion}

The preponderance of uterine fibroids in pregnancy in our practice coupled with its morphological changes due to pregnancy hormones and degeneration, which are similar to ultrasound findings with ovarian malignancies, predispose to occasional misdiagnosis. While ovarian malignancies in pregnancy are rare, early diagnosis and appropriate treatment ensure better materno-foetal-neonatal outcomes. A high index of suspicion for ovarian malignancies in pregnancy, coupled with complementing MRI (when available) with ultrasound to differentiate degenerating uterine fibroids is recommended. Since treatment is not yet standardised due to small numbers of managed ovarian malignancies in pregnancy, there is need to continue reporting our experiences managing ovarian malignancies in pregnancy, especially in the West African subregion.

\section{Declarations}

Ethics approval: Not applicable

Consent for publication: Patient gave consent

Availability of data and materials: not applicable

Competing interests : Nil

Funding : Nill 
Authors contribution: AA and MI wrote the initial draft of this article. WK and ME made major contributions to the initial draft. All authors read and approved the final manuscript.

Acknowledgement: Nil

\section{References}

1. Mancanri R, Tomasi-Cont N, Sarno NA, et al. Treatment options for pregnant women with ovarian tumours. Int J Gynecol Cancer. 2014;24(6):967-72.

2. Van Calsteren K, Heyns L, De Smet F, Van Eycken L, Gziri MM, Van Gemert W, Halaska M, Vergoye I, Ottevanger N, Amant F. Cancer during pregnancy: Analysis of 215 patients emphasising the obstetrical and neonatal outcomes. J Clin Oncol. 2010;28:683-9.

3. Leiserowitz GS, Xing G, Cress R, Brahmbhatt B, Dahrymple JL. Adnexal masses in pregnancy: how often are they malignant? Gynecol Oncol. 2006;101:315-21.

4. Amant F, Berreiller P, Boere IA, Cardonick E, Fruscio R, et al. Gynecological cancers in pregnancy: guidelines bases on a third international consensus meeting. Ann Oncol. 2019;30(10):1601-12.

5. Aggarwal P, Kehoe S. Ovarian tumours in pregnancy: a literature review. Eur J Obstet Gynecol Reprod Biol. 2011;155(2):119-24.

6. Dluski DF, Mierzynski R, Poniedzialek-Czajkowska E, Leszczynska-Gorzelak B. Ovarian cancer and pregnancy - A current problem in perinatal medicine: A comprehensive review. Cancers (Basel). 2020;12(12):3705.

7. Xu T, Wang L, Jia Y, et al. Long-term multidisciplinary integrative therapy management resulted in favourable outcomes for ovarian cancer during pregnancy: a case report and literature review. $J$ Ovarian Res, 2019; 12(108): doi:10.1186.

8. Keijser J, Sayasneh A, Van Hord K. Presurgical diagnosis of adnexal tumours using mathematical models and scoring systems: a systemic review and meta-analysis. Human Reprod Update. 2014;20:449-62.

9. Han J de, Verheecke M, Amant F. Management of ovarian cysts and cancer in pregnancy. Facts Views Vis Obgyn. 2015;7(1):25-31.

10. Patenande $Y$, Pugash D, Lim M, Marin L. The use of magnetic resonance imaging in obstetric patients. J Obstet Gynaecol Can 2014: 36(4): 349-355.

11. Forstner R, Thomassin-Naggara I, Cunha TM, Kinkel K, Masselli G. ESURE recommendations for MRI imaging of the sonographically indeterminate adnexal mass; an update. Eur Radiol. 2017;27:224857.

12. Anthoulakis C, Nikobudis N. Pelvic MRI as a 'gold standard' in the subsequent evaluation of ultrasound-indeterminate adnexal lesions: A systematic reiew. Gyn Oncol. 2014;132(3):661-8.

13. Fermung LEP, Jones K, Mas A, Kleven D, Wailer JL, Al-Hendy A. Expanding upon the human myometrial stem cell hypothesis and the role of race, hormones, age and parity in a profibroid 
environment. Am J Pathol. 2018;188(10):2293-306.

14. Awoleke JO. Caesarean nyomectomy - An option in fibroid-endemic, low-resource setting? Trop J Obstet Gynaecol. 2012;29(1):60-5.

15. Lee HJ, Norwitz ER, Shaw J. Contemporary management of fibroids in pregnancy. Rev Obstet Gynecol. 2010;3(1):20-7.

16. Vitale SG, Tropea A, Rossetti D, Cornelli M, Cranci A. Management of uterine leiomyomas in pregnancy: review of literature. Update Surg. 2013;65:179-82.

17. Omigbodun AO, Fawole AO. Myomectomy during pregnancy and delivery: Is it safe? Commentary. Trop J Obstet Gynaecol. 2005;22(1):1-3.

18. Aziken ME, Osaikhuwuomwan JA, Osemwenkha AP, Olokor OE, Iribhogbe I, Uwagboe CU. Pregnancies complicated with uterine fibroids: A case series on myomectomy in early pregnancy. Trop J Obstet Gynaecol. 2017;34:160-4.

19. Ehigiegba AE, Ande AB, Obobo SI. Myomectomy at caesarean section. Int J Gyn Obstet. 2001;75:215.

20. Ande ABA, Ehigiegba AE, Umeora OUJ. Repeat myomectomy at caesarean section. Arch Gynecol Obstet. 2004;270:296-8.

21. Ngene NC, Moodley T. Postpartum haemorrhage from a large uterus with concealed fibroids. J Obstet Gynaecol. 2014;34(5):442-3.

22. Cordeiro CN, Gemignani ML. Gynecologic malignancies in pregnancy: Balancing foetal risks with oncologic safety. Obstet Gynecol Surv. 2017;72(3):184-93.

23. Leiserowitz GS. Managing ovarian masses during pregnancy. Obstet Gynecol Surv. 2006;61:463-70.

24. Grimm D, Woelber L, Trillsch F. Clinical management of epithelial ovarian cancer during pregnancy. Eur J Cancer. 2014;50(5):963-71.

25. Wang L, Huang S, Sheng X, Ren C, Wang Q, et al. Malignant ovarian tumours during pregnancy: a multicentre retrospective analysis. Cancer Management Research. 2020;12:10841-8.

26. Salani R, Billingsley CC, Crafton SM. Cancer and pregnancy: an overview for obstetricians and gynaecologists. Am J Obstet Gynecol. 2014;211(1):7-14.

27. Mukhopadhyay A, Shinde A, Naik R. Ovarian cysts and cancer in pregnancy. Best Pract Res Clin Obstet Gynecol. 2016;33:58-72.

28. Vandenbroucke T, Verheecke M, Fumagalli M. Effect of cancer treatment during pregnancy on fetal and child development. Lancet Child Adolesc Health. 2017;1:302-10.

29. Marret $H$, Lhomme $C$, Lecuru F, Canis $M$, Leveque J, et al. Guidelines for the management of ovarian cancer during pregnancy. Eur J Obstet Gynecol Reprod Biol. 2010;149:18-21.

\section{Figures}




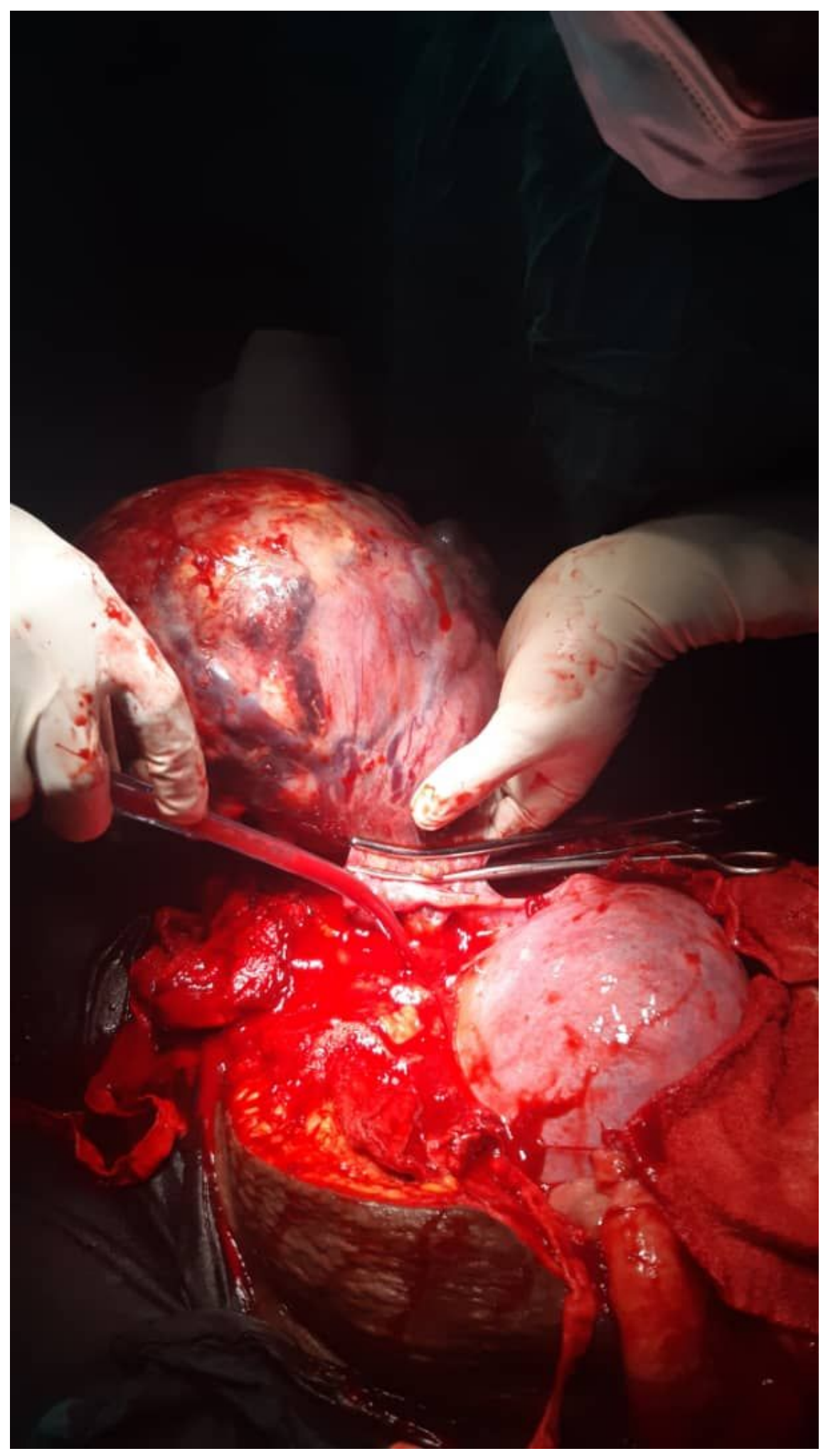

Figure 1

Excision of ovarian mass 


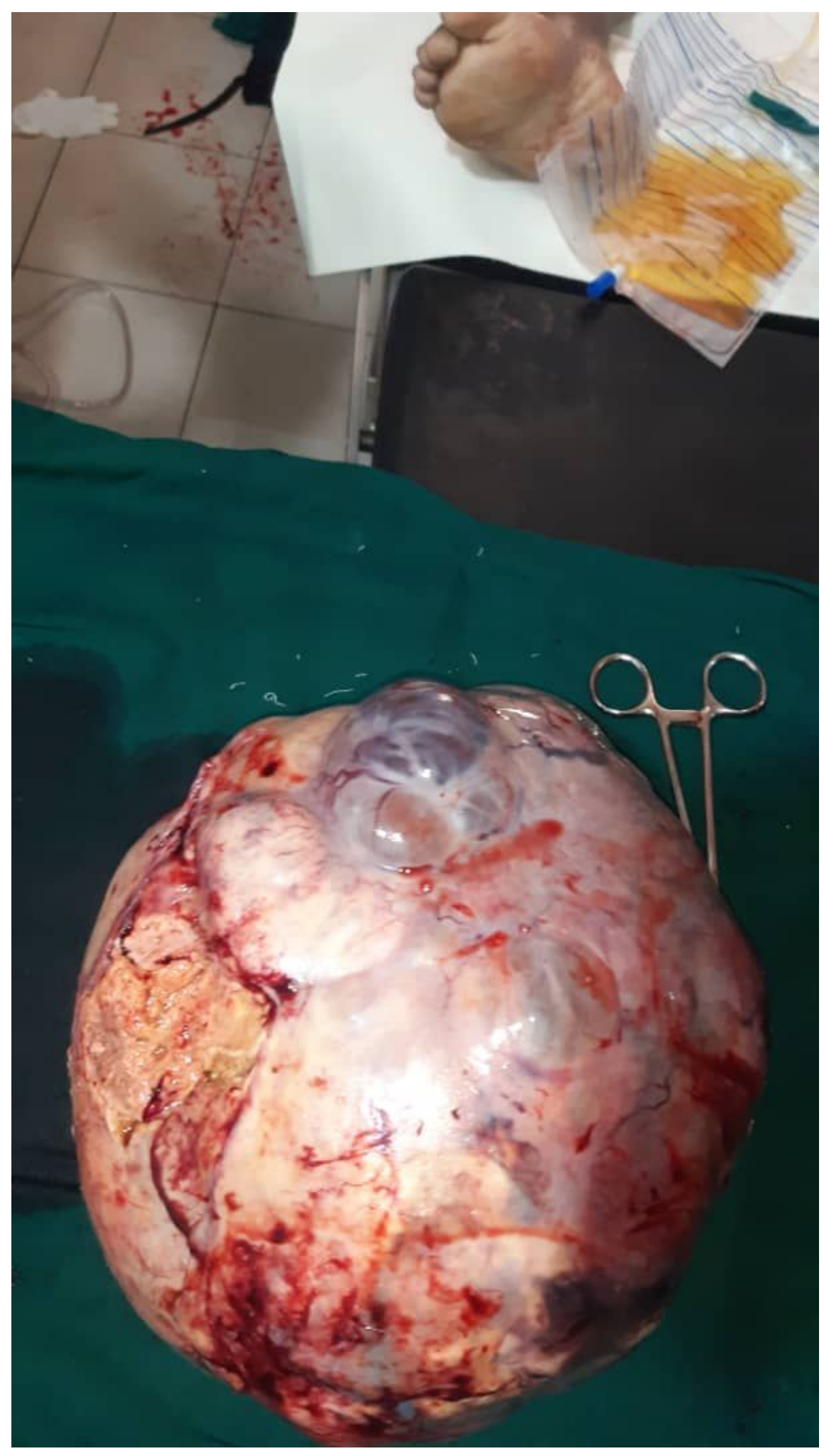

\section{Figure 2}

Right ovarian mass, $30 \times 25 \mathrm{~cm}$ (weighing $4.5 \mathrm{~kg}$ ) with cystic and solid components. 